\title{
Erratum
}

\section{PMA-Silica Gel Catalyzed Propargylation of Aromatic Compounds with Arylpropargyl Alcohols under Solvent-Free Conditions}

Pabbaraja Srihari,* Joolakanti Shyam Sunder Reddy, Satadru Sekhar Mandal, Kamani Satyanarayana, Jhillu Singh Yadav Synthesis 2008, 1853.

In the advance online (e-First) publication of this manuscript, the structure of compound 1e in Table 4 was incorrect. This mistake has been corrected for both the print and the current online versions. 